\title{
$1 \quad$ Targeting riboswitches with synthetic small RNAs for metabolic engineering
}

2

3 Milca Rachel da Costa Ribeiro Lins ${ }^{1^{*}}$, Laura Araujo da Silva Amorim ${ }^{{ }^{*}}$, Graciely Gomes

4 Correa $^{1}$, Bruno Willian Picão ${ }^{1}$, Matthias Mack², Marcel Otávio Cerri ${ }^{1}$, Danielle Biscaro

5 Pedrolli ${ }^{1 \dagger}$

6

7 'Universidade Estadual Paulista (UNESP), School of Pharmaceutical Sciences,

8 Department of Bioprocess Engineering and Biotechnology, Rodovia Araraquara-Jau

9 km1, 14800-903 Araraquara, Brazil

$10{ }^{2}$ Mannheim University of Applied Sciences, Institute for Technical Microbiology, Paul-

11 Wittsack-Str. 10, 68163 Mannheim, Germany

12

$13{ }^{*}$ Contributed equally as first author

$14{ }^{\dagger}$ Corresponding author:

15 Danielle B. Pedrolli, e-mail: danielle.pedrolli@unesp.br

16 


\section{Abstract}

18 Our growing knowledge of the diversity of non-coding RNAs in natural systems and our

19 deepening knowledge of RNA folding and function have fomented the rational design of

20 RNA regulators. Based on that knowledge, we designed and implemented a small RNA

21 (sRNA) tool to target bacterial riboswitches and activate gene expression. The synthetic

22 sRNA is suitable for regulation of gene expression both in cell-free and in cellular

23 systems. It targets riboswitches to promote the antitermination folding regardless the

24 cognate metabolite concentration. Therefore, it prevents transcription termination

25 increasing gene expression up to 103-fold. We successfully used sRNA arrays for

26 multiplex targeting of riboswitches. Finally, we used the synthetic sRNA to engineer an

27 improved riboflavin producer strain. The easiness to design and construct, and the fact

28 that the riboswitch-targeting sRNA works as a single genome copy, make it an attractive

29 tool for engineering industrial metabolite-producing strains.

32 Keywords: small RNA, non-coding RNA, riboswitch, Bacillus subtilis, synthetic biology, 33 riboflavin. 
bioRxiv preprint doi: https://doi.org/10.1101/2021.06.21.449321; this version posted June 21, 2021. The copyright holder for this preprint (which was not certified by peer review) is the author/funder, who has granted bioRxiv a license to display the preprint in perpetuity. It is made available under aCC-BY-NC-ND 4.0 International license.

\section{Graphical Abstract}

36

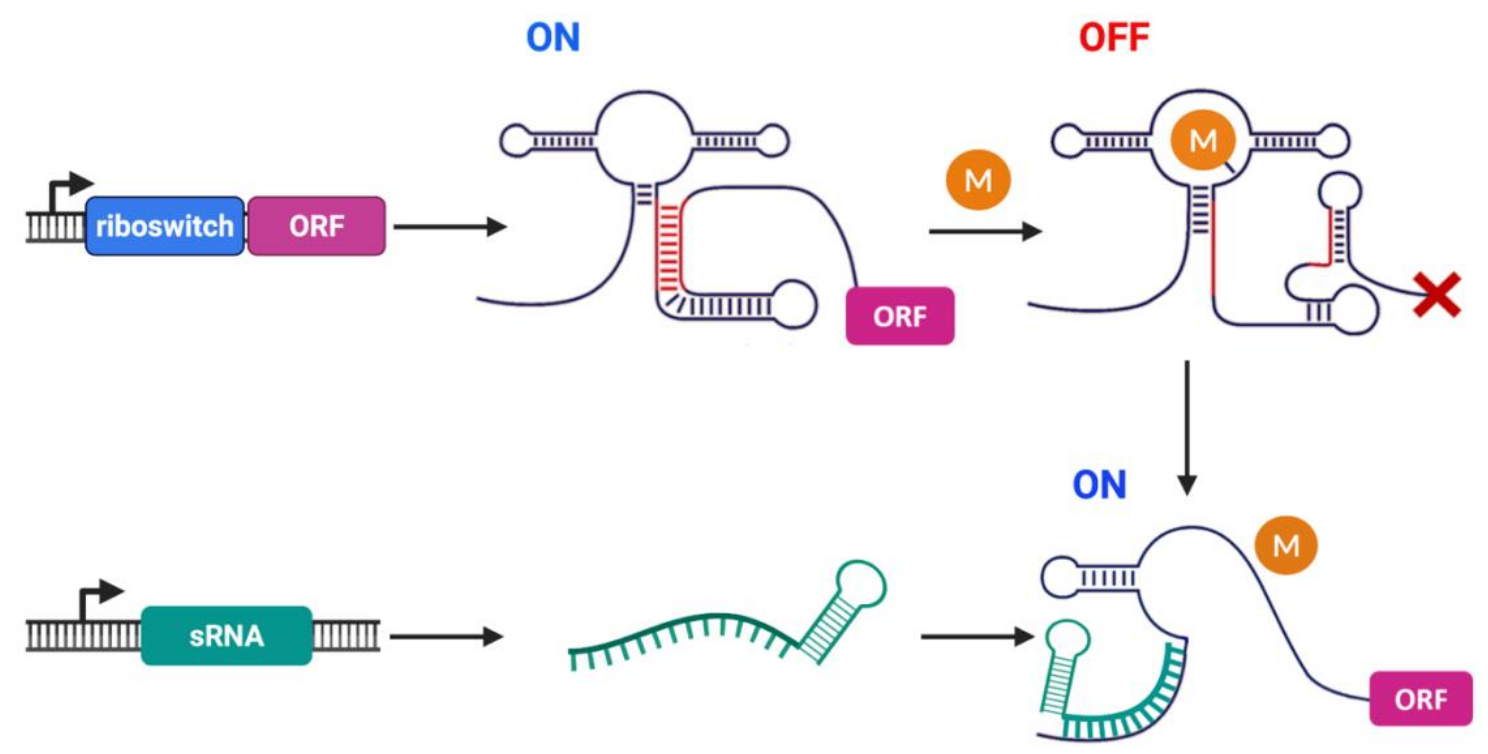

37 


\section{Introduction}

RNAs are important regulatory tools in the synthetic biology toolbox for controlling gene expression and constructing synthetic gene networks (Chappell et al., 2015b; Leistra et al., 2019). Among all regulatory RNAs, trans-acting antisense small RNAs (sRNAs) are particularly attractive to engineer due to their simplicity, fast response to external signals, and usefulness for fine-tuning of gene expression (Shimoni et al., 2007). The simplicity of the RNA sequence and the predictability of RNA folding has powered the development of sRNA rational design to tune gene expression. Moreover, the specificity of basepairing and easiness to construct make sRNA an ideal tool for circuit design and multiplex control (Kelly et al., 2018; Noh et al., 2017). Compared to protein-based regulation, sRNAs offer multiple options for the design of orthogonal control with minimal or no metabolic burden associated. Therefore, synthetic sRNAs are simple and versatile tools to regulate gene expression and engineer metabolic pathways.

Synthetic sRNAs have been mostly used as antisense RNAs to block the access of the ribosome to the mRNA and prevent translation in E. coli. sRNA-mediated knockdown has been successfully used to fine-tune gene expression and develop $E$. coli superproducers of industrially relevant compounds such as tyrosine, proline, cadaverine, putrescine, 4-hydroxycoumarin, resveratrol, and naringenin (Na et al., 2013; Noh et al., 2017; Yang et al., 2015). The strategy has been once used in B. subtilis to engineer a $\mathrm{N}$-acetylglucosamine producer strain (Liu et al., 2014). Now, we expand the functionality of sRNAs to target riboswitches and activate gene expression at the transcription level.

60 Riboswitches play an important role in the regulation of gene expression in bacteria. In B. subtilis there are 41 identified riboswitches that regulate $\sim 2 \%$ of all genes, many of them related to the biosynthesis of industrially relevant compounds (Kalvari et al., 2021; Mandal et al., 2003). Bacterial riboswitches ultimately regulate the levels of metabolites in the cell through the control of biosynthesis and/or transport processes (Mandal et al., 2003; Mars et al., 2016). Although tempting, deletion of riboswitches aiming to create 
67 al., 2020; Shi et al., 2014). Alternatively, we propose the use of riboswitch-targeting

68 sRNAs for dynamic control of gene expression.

69 Riboswitches that bind organic small molecules, such as purines, vitamins, and amino acids, keep the cell homeostasis preventing unneeded accumulation of cellular compounds. In B. subtilis and other bacteria the control exerted by riboswitches is most pronounced during the exponential growth phase when the intense cell metabolism favors biosynthetic processes (Lins et al., 2021; Pedrolli et al., 2015, 2012). The developed riboswitch-targeting sRNAs act at that time point, interfering with the riboswitch aptamer folding, preventing transcription termination, and stabilizing the mRNA, which leads to metabolite accumulation.

\section{Materials and methods}

\section{1. sRNA design analysis in silico}

The synthetic RNAs were designed for each riboswitch using the RiboMaker software

81 (Rodrigo and Jaramillo, 2014; Rostain et al., 2015). The thermodynamic properties of the sRNAs were evaluated using the NUPACK Web Application (Zadeh et al., 2011).

\subsection{Plasmids and other DNA sequences}

85 Plasmids for riboswitch control of gene expression have been constructed previously 86 (Lins et al., 2021). Shortly, for in vitro gene expression each riboswitch was cloned between the $E$. coli ribB promoter and the firefly luciferase gene $(I u c)$ to generate plasmid series $\mathrm{P}_{\text {ribB-riboswitch-luc. For in vivo gene expression, expression each riboswitch was }}$ cloned between the $\mathrm{P}_{\text {srf }}$ promoter and the luxABCDE operon to generate the plasmid series pBS3C-riboswitch-lux. The plasmids integrate at the sacA locus in B. subtilis.

91 The synthetic sRNAs were purchased as oligonucleotides forward and reverse strands, 92 annealed, and cloned into the pBS2E plasmid (Radeck et al., 2013) together with the $93 \mathrm{P}_{\text {grac }}$ promoter to generate the plasmid series pBS2E- $\mathrm{P}_{\text {grac }}-\mathrm{SRNA}$. The plasmids integrate 
94 at the lacA locus in $B$. subtilis. The $\mathrm{P}_{\text {grac }}$-SRNA-purE-broccoli sequence was cloned into

95 the pBS1C plasmid (Radeck et al., 2013) and integrated at the amyE locus in B. subtilis.

96 The sRNA operon was assembled from 28 semi-complementary oligonucleotides

97 designed with GeneDesign (Richardson et al., 2006). Four building blocks were generated by PCR gene synthesis (Rouillard et al., 2004). The four blocks were then assembled using Overlap Extension PCR. Lists of plasmids and strains used are provided in the supplementary tables S1 e S2.

\subsection{In vitro transcription}

103 RNAs transcripts were generated by in vitro transcription using the RiboMAX ${ }^{\text {TM }}$ Large

104 Scale RNA Production Systems (Promega). Annealed oligonucleotides forward and reverse containing the T7 promoter and the sRNA were used as template. Reactions were incubated at $37^{\circ} \mathrm{C}$ overnight. Reaction inactivation was performed at $70^{\circ} \mathrm{C}$ for 20

107 min. To eliminate the template, all samples were treated with RQ1 RNase-Free DNase

108 (Promega) for 1 hour at $37^{\circ} \mathrm{C}$. Samples were then precipitated with ethanol and resuspended in RNase-free water.

\subsection{In vitro gene expression}

112 The regulatory activity of the sRNAs was accessed by in vitro gene expression reaction

113 according to Lins et al. (Lins et al., 2021). Each $P_{\text {ribB-riboswitch-luc plasmid was extracted }}$

114 from overnight grown E. coli with a miniprep kit (PureYield ${ }^{\mathrm{TM}}$ Plasmid Miniprep System,

115 Promega) and purified using ethanol precipitation. Plasmids were resuspended in

116 RNase-free water and used as templates for in vitro gene expression (E. coli S30 Extract

117 System for Circular DNA, Promega). Reactions were set up by adding $1 \mu \mathrm{L}$ of $0.25 \mathrm{mM}$ 118 amino acid mix, $4 \mu \mathrm{L}$ of S30 Premix, $3 \mu \mathrm{L}$ of S30 Extract, $1 \mu \mathrm{L}$ of $50 \mu \mathrm{M}$ ligand (guanine, 119 adenine or FMN; Merck), $1 \mu \mathrm{L}$ of transcribed sRNA (30 $\mathrm{ng} \mu \mathrm{L}^{-1}$ ), and $1 \mu \mathrm{L}$ of plasmid (5 $\left.120 \mathrm{ng} \mu \mathrm{L}^{-1}\right)$. The reaction mixture was incubated at $30^{\circ} \mathrm{C}$ for $20 \mathrm{~min}$, and $90 \mu \mathrm{L}$ of $1 \%(\mathrm{~m} / \mathrm{v})$ 
121 bovine serum albumin was added to stop the reactions. Each treatment was performed

122 in triplicate.

123 In order to quantify the luciferase activity, $10 \mu \mathrm{L}$ of stopped reactions were added to 50

$124 \mu \mathrm{L}$ of luciferase substrate solution (Luciferase Assay System kit, Promega) in a 96-well 125 white flat bottom microplate. Luminescence readings were taken on a microplate reader

126 (Tecan Infinite 200 Pro, Switzerland) set with 1,000 ms for integration time at $26^{\circ} \mathrm{C}$.

\subsection{Bacterial strains and media}

129 E. coli Top10 was used for cloning and propagation of plasmids. It was aerobically

130 cultivated at $37^{\circ} \mathrm{C}$ in Lysogeny Broth (LB) enriched with antibiotics when needed. $B$.

131 subtilis 168, B. subtilis riboflavin producer (BsRF), and B. subtilis Ai (BsAi) (Correa et al.,

132 2020) were used as chassis for the experiments as indicated in the main text. All $B$.

133 subtilis strains were aerobically cultivated in LB for all experiments regarding

134 bioluminescence measurements. A high sugar complex medium was used for riboflavin 135 production in all scales (PW medium: $40 \mathrm{~g} \mathrm{~L}^{-1}$ sucrose, $1 \mathrm{~g} \mathrm{~L}^{-1}$ yeast extract, $25 \mathrm{~g} \mathrm{~L}^{-1}$ $136 \mathrm{NaNO}_{3}, 0.333 \mathrm{~g} \mathrm{~L}^{-1}, \mathrm{KH}_{2} \mathrm{PO}_{4}, 1 \mathrm{~g} \mathrm{~L}^{-1} \mathrm{Na}_{2} \mathrm{HPO}_{4} \cdot 12 \mathrm{H}_{2} \mathrm{O}, 0.15 \mathrm{~g} \mathrm{~L}^{-1} \mathrm{MgSO} 4 \cdot 7 \mathrm{H}_{2} \mathrm{O}, 7.5 \mathrm{mg}$

$137 \mathrm{~L}^{-1}, \mathrm{CaCl}_{2}, 6 \mathrm{mg} \mathrm{L}-1 \mathrm{MnSO} 4 \cdot \mathrm{H}_{2} \mathrm{O}, 6 \mathrm{mg} \mathrm{L}^{-1}, \mathrm{FeSO}_{4} 7 \mathrm{H}_{2} \mathrm{O}, \mathrm{pH}$ 7.0). B. subtilis culture 138 medium was enriched with chloramphenicol $\left(5 \mu \mathrm{g} \mathrm{mL}^{-1}\right)$ and/or erythromycin $\left(1 \mu \mathrm{g} \mathrm{mL}^{-1}\right)$ 139 when needed.

142 Bioluminescence measurements from growing B. subtilis were carried out in white 96143 well microplates with optically clear bottoms. Culture density was monitored by 144 absorbance measurements at $600 \mathrm{~nm}\left(\mathrm{OD}_{600}\right)$ taken from the same transparent bottom 145 microplates. Plates were incubated at $37^{\circ} \mathrm{C}$ and orbital shaking at $143 \mathrm{rpm}$. 146 Measurements were performed every $10 \mathrm{~min}$ for five hours in the microplate reader 147 (Tecan Infinite 200 Pro). All experiments were performed with three biological replicates. 


\subsection{Broccoli-DFHBI fluorescence measurements}

150 The RNA-Broccoli (Filonov et al., 2014) was fused to the purE-sRNA under control of the

$151 \mathrm{P}_{\text {grac }}$ for detection of transcripts in B. subtilis. After induction with IPTG, samples were 152 withdrawn and incubated for $30 \mathrm{~min}$ with the fluorophore DFHBI. Then, fluorescence 153 measurements were taken in a 96-well black microplate with the microplate reader set 154 at $472 \mathrm{~nm}$ for excitation and $507 \mathrm{~nm}$ for emission.

\subsection{Test tube and Erlenmeyer scale cultivation}

157 B. subtilis cultivation was performed in $16 \times 220 \mathrm{~mm}(\mathrm{~d} \times \mathrm{L})$ test tubes filled with $5 \mathrm{~mL}$

158 culture medium and incubated at $37^{\circ} \mathrm{C}$ and $220 \mathrm{rpm}$. B. subtilis culture was also carried 159 out in $250 \mathrm{~mL}$ baffled Erlenmeyer flasks filled with $20 \mathrm{~mL}$ culture medium and incubated 160 at $37^{\circ} \mathrm{C}$ and $150 \mathrm{rpm}$. Samples were periodically withdrawn for absorbance 161 measurements at $600 \mathrm{~nm}\left(\mathrm{OD}_{600}\right)$ in a microplate reader (Tecan Infinite 200 Pro,

162 Switzerland), and for riboflavin quantification by HPLC. All experiments were performed 163 with three biological replicates.

\subsection{Bioreactor scale cultivation}

166 A stirred batch was carried out at $37^{\circ} \mathrm{C}, 0,5 \mathrm{vvm}$ air supply, and $\mathrm{pH}$ controlled at $7.0 \pm 0.1$.

167 The bioreactor $7 \mathrm{~L}$ tank was filled with $5 \mathrm{~L}$ of PW medium supplemented with $80 \mathrm{~g} \cdot \mathrm{L}^{-1}$ 168 sucrose. After inoculation, samples were withdrawn periodically for sucrose, riboflavin, 169 and biomass quantification. Biomass quantification was accessed as dry weight per liter.

\subsection{Riboflavin and sucrose quantification}

172 Samples withdrawn from cultures were centrifuged at $16,000 \mathrm{xg}$ for $30 \mathrm{~min}$ for 173 supernatant recovery. Trichloroacetic acid $1 \%(\mathrm{v} / \mathrm{v})$ was added to the supernatant to 174 precipitate proteins. The resulting samples were centrifuged at $16,000 \times \mathrm{gg}$ for $20 \mathrm{~min}$. The 175 supernatant was then filtered using a hydrophilic PTFE $0.20 \mu \mathrm{m}$ membrane, and the 
176 filtrate was used for analysis by high-performance liquid chromatography (HPLC;

177 Shimadzu LC-20AD equipped with a SPD-M20A photodiode detector).

178 Riboflavin was analyzed using a $50 \times 3 \mathrm{~mm}$ Poroshell 120 EC-C18 column (Agilent

179 Technologies) in $82 \%$ solvent $\mathrm{A}$ ( $20 \mathrm{mM}$ formic acid, $20 \mathrm{mM}$ ammonium formate) and

$18018 \%$ solvent $\mathrm{B}$ (methanol) as mobile phase at $0.5 \mathrm{~mL} / \mathrm{min}$, in isothermal mode at $30^{\circ} \mathrm{C}$.

181 Detection was performed at $445 \mathrm{~nm}$. Commercial riboflavin (Merck) was used as

182 standard for calibration.

183 Sucrose was analyzed using a HPX-87P column (Aminex) in ultrapure water as mobile 184 phase at $0.6 \mathrm{~mL} / \mathrm{min}$, in isothermal mode at $80^{\circ} \mathrm{C}$.

\subsection{Statistical analysis}

187 Analysis of variance (ANOVA) and Tukey test were performed using the Minitab software

188 (version 18), to compare the averages and observe the significance of the results.

\section{Results}

\subsection{Designing small regulatory RNAs to target riboswitches}

We have chosen the well-studied purine riboswitches purE, xpt, nupG, and pbuE, and the flavin riboswitch ribDG of $B$. subtilis as targets for sRNA design. Together they control

194 the purine uptake and synthesis and the riboflavin synthesis through premature

195 transcription termination (Johansen et al., 2003; Lins et al., 2021; Mandal et al., 2003).

196 The sRNA was designed to target each riboswitch in order to prevent folding into the

197 OFF structure that leads to premature transcription termination (Figure 1). The sRNA 198 was designed to counteract the riboswitch activity and keep gene expression ON even 199 at high cognate metabolite concentration by promoting the anti-terminator formation even when it shouldn't. Target sequences in the aptamers have been previously

201 identified through point mutation as crucial for the OFF state folding (Mandal and

202 Breaker, 2004; Marcano-Velázquez and Batey, 2015; Mironov et al., 2002). sRNA 203 sequences targeting each riboswitch were generated using RiboMaker (Rodrigo and 
204 Jaramillo, 2014) and analyzed using NUPACK (Zadeh et al., 2011). The best sRNAs

205 were selected based on the hybridization free energy (lower than $-18 \mathrm{Kcal} / \mathrm{mol}$ ), the 206 seed-based free energy, ensemble defect (lower than 10\%), and the complex 207 representation at equilibrium (Rostain et al., 2015). One sRNA targeting each riboswitch was selected for construction and test (Table S3).

209

\section{A) xpt riboswitch}

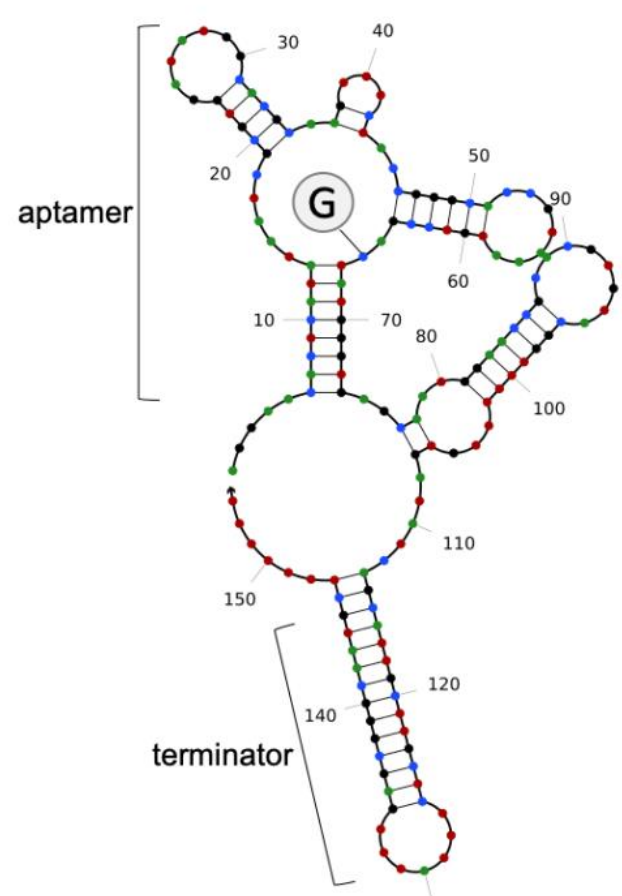

130

\section{B) $x p t$ riboswitch-sRNA}

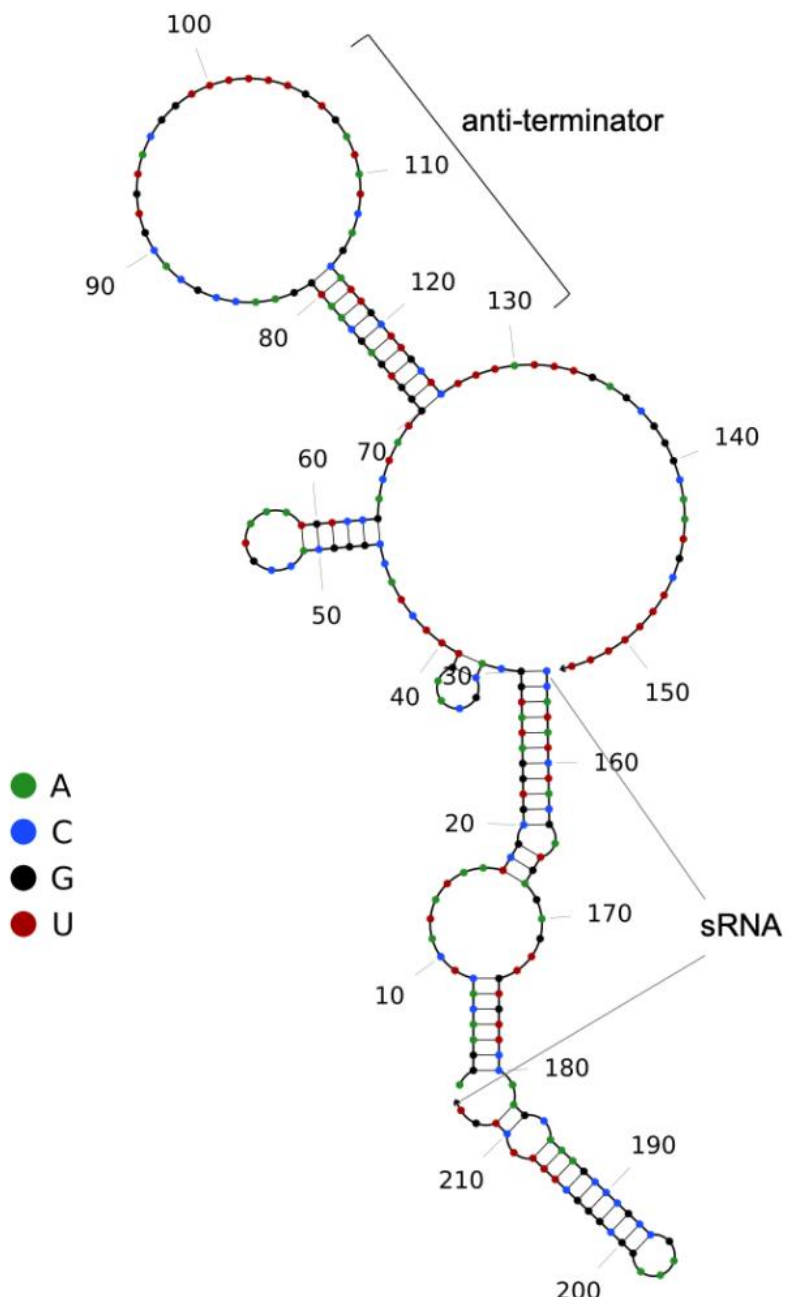

Figure 1. Riboswitch-sRNA paring. (A) sequence and structure of the xpt riboswitch bound to

212 guanine. (B) sequence and structure of the xpt riboswitch paired with the xpt-sRNA. sRNA paring

213 with the riboswitch nucleotides 2-30 results in an alternative folding, that leads to the formation of

214 the anti-terminator steam loop through base-pairing between nucleotides $70-81$ and $115-126$. All

215 riboswitch-targeting sRNAs were designed to target the aptamer sequence in order to promote

216 the anti-terminator formation. Figure generated using NUPACK Web Application. 

vitro

219 In order to evaluate the specific ability of each synthetic sRNA to bind its target riboswitch

220 and activate gene expression, we set an in vitro gene expression reaction which has

221 been validated for riboswitch characterization (Lins et al., 2021). Expression of the firefly

222 luciferase gene under the control of each riboswitch was evaluated in the presence of 223 the sRNA and the cognate metabolite (guanine, adenine, or FMN). Each sRNA was

224 previously transcribed in vitro and purified. All sRNAs were able to completely counteract

225 their target riboswitch's regulatory activity turning ON gene expression when it was

226 supposed to be OFF (Figure 2). The activation fold ranged from 2.1 to 4.2. Interestingly,

227 the sRNAs purE, pbuE, and ribDG activated gene expression to levels above the

228 riboswitches' ON state (Figures 2A, D, and E). The sRNA effect on the riboswitch was

229 compared to control-sRNAs, which are riboswitch-targeting sRNAs tested against non-

230 target riboswitches. 


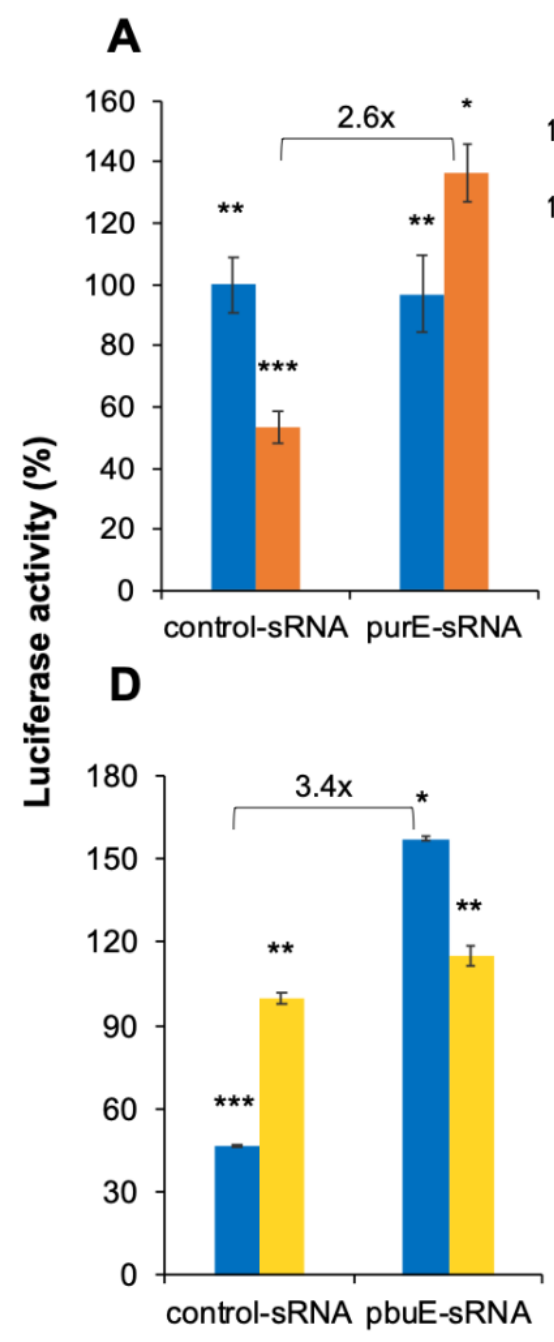

B

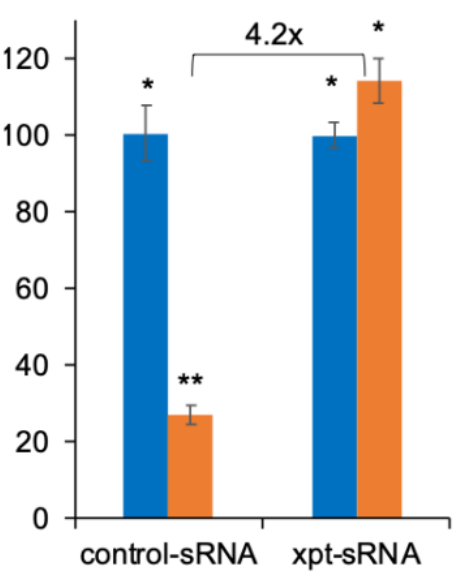

$\mathbf{E}$

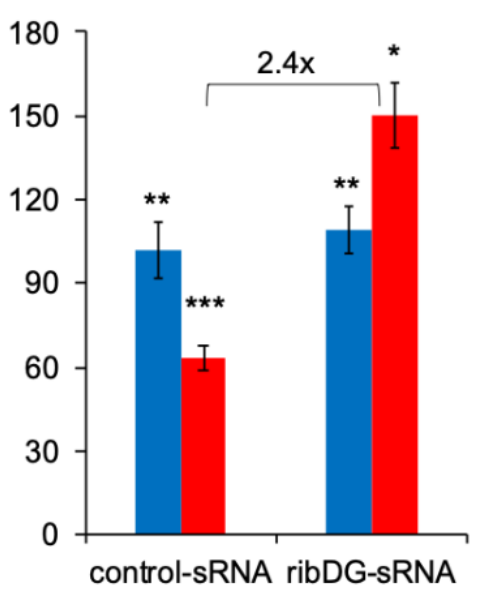

C

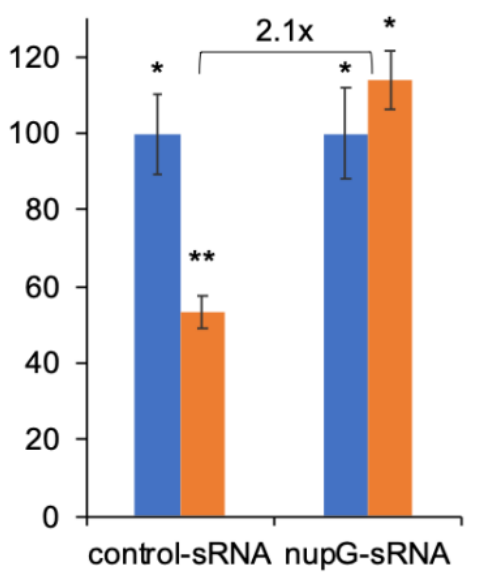

$\square$ No additive

$\square$ Guanine

$\square$ Adenine

FMN

234 Figure 2. sRNA activity and specificity assayed by in vitro expression of the firefly luciferase gene.

235 (A) purE riboswitch. (B) xpt riboswitch. (C) nupG riboswitch. (D) pbuE riboswitch. (E) ribDG 236 riboswitch. The sRNA effect on the riboswitch was compared to control-sRNAs, which are 237 riboswitch-targeting sRNAs tested against non-target riboswitches. Asterisks identify statistically 238 significant differences $(p<0.05)$. Data are presented as mean $\pm \operatorname{SD}(n=3)$. Raw data available 239 in Supplementary Data 1.

\subsection{Synthetic sRNAs are transcribed and functional in the cell}

242 After confirming that each synthetic sRNA can specifically counteract the target

243 riboswitch in vitro, we moved to $B$. subtilis to verify whether the sRNA could be stably 244 transcribed in vivo. Therefore, the purE-sRNA was fused to the broccoli sequence at the 3'-end and integrated as single-copy to the chromosome. Expression of the purE-sRNA- 
246 broccoli was induced with IPTG in the early-exponential phase, and samples were taken

247 for DFHBI-broccoli fluorescence measurements. A fluorescence peak was detected $1 \mathrm{~h}$

248 post-induction and fluorescence was still detectable after 24h (Figure 3A).

249

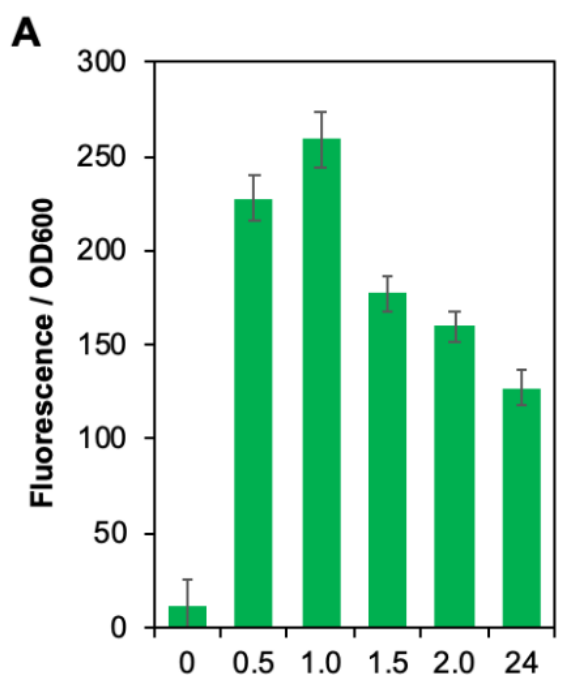

250

Post-induction incubation (h)

B

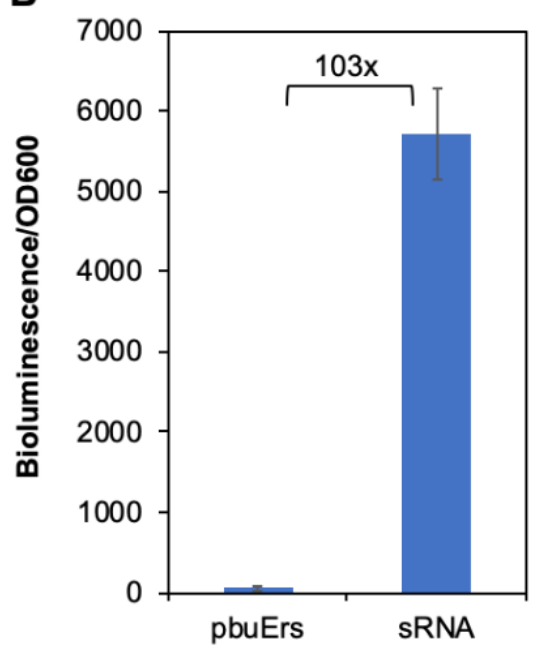

C

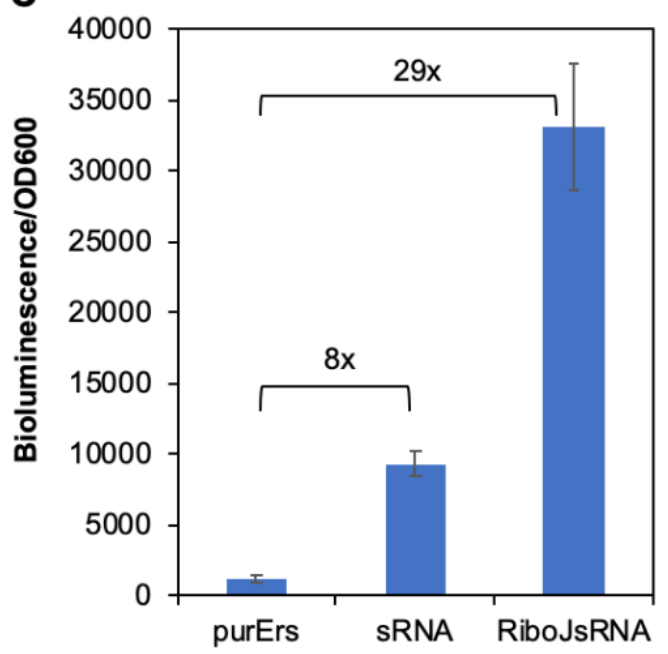

253 Figure 3. sRNA expression and activity assayed in B. subtilis. (A) sRNA-broccoli-DFHBI fluorescence. (B) the lux operon under control of the pbuE riboswitch (pbuErs) and the pbuE-

255 sRNA (sRNA) activation effect on the riboswitch. (C) the lux operon under control of the purE 256 riboswitch (purErs) and the pbuE-sRNA (sRNA) and the RiboJ-purE-sRNA (RiboJsRNA) 257 activation effect on the riboswitch. Data are presented as mean $\pm \operatorname{SD}(n=3)$. Raw data available in Supplementary Data 2. 
260 After validating the ability of $B$. subtilis to stably transcribe the sRNA, we constructed a

261 reporter assay to access the sRNA activity in vivo. Therefore, the luxABCDE operon was used as a reporter under the control of the riboswitches purE and pbuE. Each riboswitchlux construct was inserted as single-copy at the locus sacA, and the corresponding sRNA was integrated at the lacA locus in the chromosome. The sRNA transcription was driven by the strong $\mathrm{P}_{\text {grac }}$ promoter working constitutively (the strain lacks a lacl gene). The resulting strains were cultivated in Erlenmeyer flasks filled with LB medium without additives and monitored for bioluminescence for $8 \mathrm{~h}$. The pbuE-sRNA showed a strong activity activating gene expression up to 103-fold (Figure 3B), much higher than observed in vitro. The purE-sRNA caused an 8-fold increase in gene expression (Figure 3C), which is three times higher than achieved in vitro. Although higher than in vitro, gene the purE-sRNA activating activity in vivo is much lower than the activity measured for the pbuE-sRNA. We hypothesized that the purE-sRNA lower activity could be caused by low RNA stability in the cell. Therefore, we fused it to the RiboJ ribozyme to create the RiboJpurE-sRNA. RiboJ greatly improved the sRNA, causing a 29-fold increase in gene expression (Figure 3C). RiboJ is a structured RNA able to self-cleave at the 5'-end that has been used to insulate mRNAs (Lou et al., 2012). Any other structured RNA could have had the same beneficial effect on the sRNA; however, a self-cleaving ribozyme

278 offers the possibility to build an sRNA array for targeting multiple loci (multiplex control).

279 Noteworthy, the sRNAs pbuE and RiboJpurE improved the strain growth significantly compared to the control strains carrying the riboswitch-controlled lux operon but no sRNA (Figure S1). The low-efficient purE-sRNA did not cause any change in the strain growth.

\subsection{Engineering an improved riboflavin producer strain}

Synthetic sRNA has great applicability as a tool to fine-tune gene expression in metabolic 
al., 2014; Na et al., 2013; Noh et al., 2017; Sun et al., 2019; Yang et al., 2019, 2015). In order to demonstrate the potential of the riboswitch-targeting sRNA for metabolic engineering, we tested the sRNA ability to improve the riboflavin production directly by targeting the rib operon, and indirectly by targeting the purine supply (Figure 4). The sRNAs ribDG, purE, RiboJpurE, xpt, and pbuE were individually inserted into the

292 genome of a riboflavin super-producing strain (BsRF) as single copies. Riboflavin 293 production was first evaluated in test tubes (Figure 5A). Although the sRNAs purE and

294 RiboJpurE have been previously proved to be functional in vivo, they did not improve the 295 riboflavin production. Both target the pur operon, which is responsible for the synthesis of inosine monophosphate (IMP) from phosphoribosyl-a-1-pyrophosphate (PRPP). IMP is the precursor for AMP, GMP, and hypoxanthine; therefore, the carbon flux may divert to other routes instead of GTP.

299 The pbuE sRNA targets the expression of the adenine exporter PbuE, increasing it.

300 Although the pbuE sRNA has proven highly functional using the lux reporter, the strain

301 carrying it produced lower levels of riboflavin (Figure 5A). Increased adenine efflux 302 probably increased the IMP consumption to reset the intracellular adenine concentration, which could cause a reduced flux through GMP synthesis.

304 The sRNAs $x p t$ and ribDG, on the other hand, were able to increase the riboflavin 305 production by $48 \%$ and $30 \%$, respectively. The xpt sRNA targets the expression of the $306 x p t-p b u X$ operon. pbuX encodes a membrane protein that imports xanthine from the 307 medium. xpt encodes a xanthine phosphoribosyl transferase, which converts xanthine to 308 xanthine monophosphate (XMP) (Christiansen et al., 1997). XMP is then converted to 309 GTP, which serves as a direct precursor to riboflavin (Figure 4). The ribDG sRNA targets 310 the ribDGBAHT operon, which encodes the enzymes directly responsible for riboflavin 311 biosynthesis from GTP and ribulose (Bacher et al., 1993). 


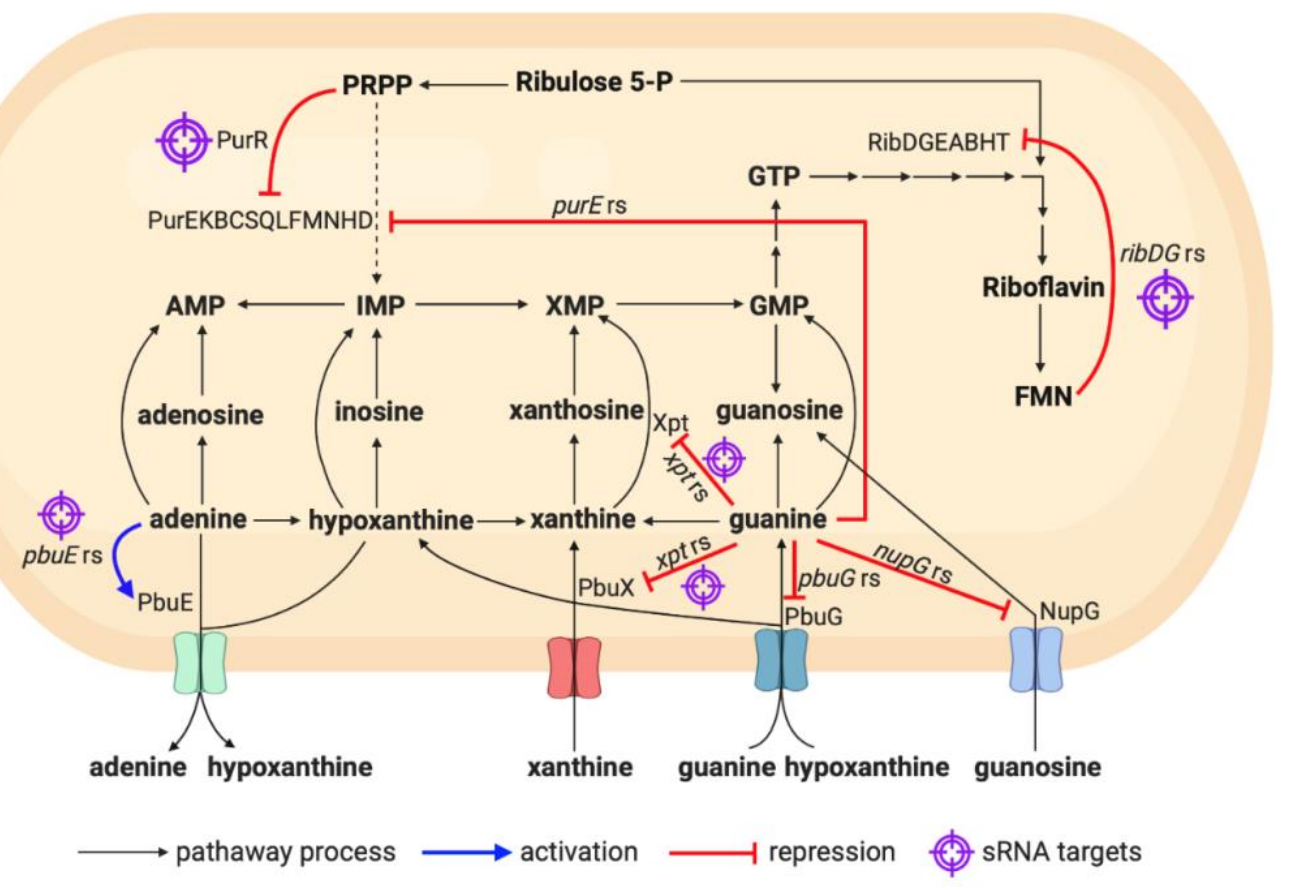

314 Figure 4. Metabolic engineering of $B$. subtilis RF (BsRF) for riboflavin production using sRNAs.

315 The riboswitches identified as purE rs, pbuE rs, $x p t r s, p b u G r s$, nupG rs, and ribDG rs regulate

316 the expression of important genes and operon from the purine and riboflavin metabolism.

318 To compare the performance of the designed riboswitch-targeting sRNAs with traditional 319 antisense sRNAs (Liu et al., 2014; Na et al., 2013) we inserted the purR-sRNA into the

320 B. subtilis chromosome. The latter was designed to pair with the purR mRNA from the

321 RBS to the +14 ribonucleotide, thereby blocking translation initiation. The antisense RNA

322 targets the synthesis of PurR, a repressor that blocks transcription initiation of several

323 genes involved in the purine metabolism including the pur operon and xpt-pbuX (Saxild 324 et al., 2001). The strain carrying the purR-sRNA produced 34\% more riboflavin than the control (Figure 5A), resembling the xpt-sRNA and ribDG-sRNA carrying strains. 
A

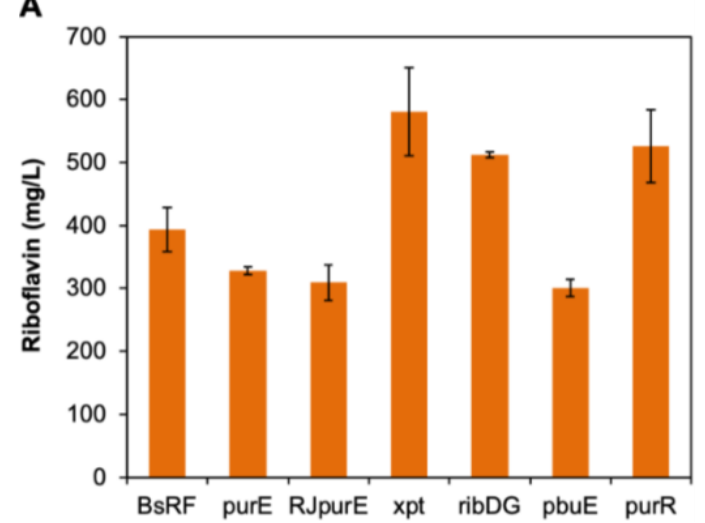

C

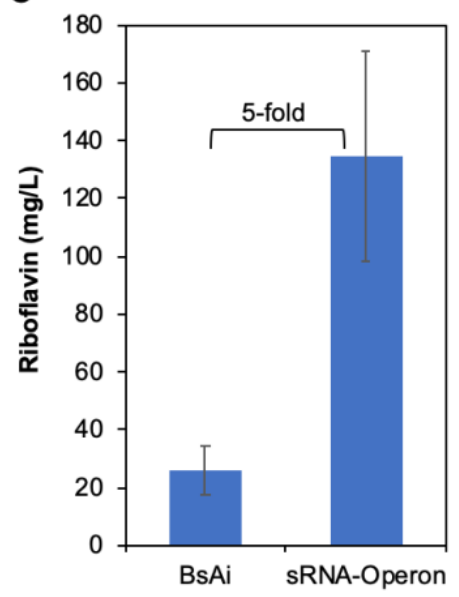

B

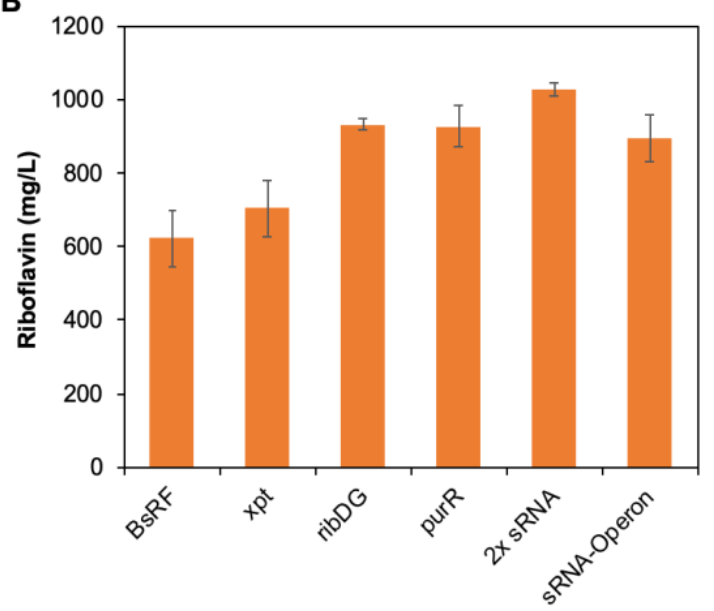

Figure 5. Engineering B. subtilis with sRNAs for riboflavin production. (A) Test tube cultivation to evaluate the effect of sRNAs on the riboflavin production. The effect of the sRNAs purE, RiboJpurE, $x p t$, ribDG, pbuE, and purR were tested in the BsRF strain. (B) Erlenmeyer cultivation of the BsRF strains carrying the best sRNAs ( $x p t$, ribDG, and purR) individually, an sRNA array composed of 2 sRNAs (ribDG and purR), and an sRNA operon composed of five sRNAs (purE, $x p t$, ribDG, pbuE, and purR). (C) The sRNA operon tested in the B. subtilis strain BsAi. Data are presented as mean $\pm S D(n=3)$. Raw data available in Supplementary Data 3.

The most promising strains, carrying the sRNAs $x p t$, ribDG, and purR, were further tested in Erlenmeyer flasks (Figure 5B). Upscaling resulted in increased riboflavin production for all tested strains. The highest production was triggered by the sRNAs ribDG and purR reaching over $900 \mathrm{mg} / \mathrm{L}$, which is about $50 \%$ higher than the control. The xpt-sRNA did not repeat the performance shown on a smaller scale and only slightly increased the 
342 riboflavin production, not significantly though. Interestingly, the strain carrying the xpt-

343 sRNA grew as poor as the riboflavin super-producing strain (BsRF), whose slow growth

344 has been ascribed to the burden of riboflavin overproduction. However, the two sRNAs

345 that increased the riboflavin production, ribDG and purR, seem to restore the growth

346 capability of the producer strain reaching cell densities around 2.5-fold higher than BsRF

347 after 40h (Figure S2).

\subsection{Multiplex sRNA for targeting riboswitches simultaneously}

350 After testing the synthetic sRNAs individually, we wondered whether they could be combined for an additive effect on the riboflavin production. We designed a sRNA array composed of the sRNAs purR and ribDG each carrying its own promoter and terminator.

353 Combining the two best sRNAs purR and ribDG increased $10 \%$ the riboflavin production compared to only one of them, which represents a $65 \%$ increase compared to the parental strain (Figure 5B). Next, we wanted to simultaneously test all the sRNAs developed. To avoid promoter and terminator repetition, we constructed a sRNA operon under control of a single promoter and terminator. The ribozymes RiboJ, RiboJ10, RiboJ60 e RiboJ64 (Nielsen et al., 2016) were inserted in between the sRNAs to promote the release of each SRNA after transcription. The sRNA operon was first tested in the

360 riboflavin super-producing strain (BsRF) resulting in a $44 \%$ increase in the riboflavin titer

361 compared to the parental strain (Figure 5B). Although functional, the sRNA operon 362 showed a smaller effect than the best sRNAs individually, which may be attributed to a 363 combination of the positive influence of some sRNAs ( $x p t$, purR, and ribDG) and the 364 negative influence of others (purE and $p b u E$ ) in the riboflavin biosynthesis. Noteworthy, both strains, the BsRF::purR-sRNA-ribDG-sRNA and the BsRF::sRNA-operon, grew to

366 higher culture densities than the parental strain BsRF, closely reproducing the effect 367 observed for the individual sRNAs ribDG and purR.

368 The BsRF strain has several mutations that increase flux through the riboflavin 369 biosynthetic pathway and increase the availability of the precursor ribulose 6 -phosphate. 
370 Therefore, we hypothesized that the sRNAs effect could be restricted in such a context.

371 In order to evaluate the full potential of the sRNA operon, we inserted it into the genome

372 of a B. subtilis strain (BsAi) that carries an extra copy of the riboflavin biosynthetic operon

373 under control of an autoinduction device (Correa et al., 2020), but no other modification.

374 The sRNA operon increased 5-fold the riboflavin production compared to the parental

375 strain (Figure $5 \mathrm{C}$ ). This result demonstrates that our synthetic sRNA operon is fully

376 functional and capable of targeting gene expression.

3.6. The BsRF::ribDG-sRNA strain is stable and highly productive in a bioreactor scale

380 In order to demonstrate that our approach is suitable for engineering industrial metabolite-producing strains that are stable and highly productive in large scale, we scaled up the cultivation to a bioreactor. Therefore, one of our best riboflavin producing

383 strain, the BsRF::ribDG-sRNA, was cultivated in a $5 \mathrm{~L}$ batch to access its potential for 384 large scale riboflavin production. The bioreactor was operated in four different conditions 385 regarding the agitation (from 200 to $800 \mathrm{rpm}$ ) and the best condition was repeated to confirm the results. Results show that slowing down the rotation to $300 \mathrm{rpm}$ favors riboflavin production over biomass formation (Table S4). Cultivation at this condition resulted in $2.1 \mathrm{~g} / \mathrm{L}$ of riboflavin after $48 \mathrm{~h}$ (Figure 6), corresponding to a yield of $44.2 \pm 5.9$ $\mathrm{mg} / \mathrm{L}$.h. The final riboflavin titer was 2.2-fold higher compared to the same strain

390 cultivated in Erlenmeyer scale. This result shows that the sRNA-carrying strain is suitable 391 for riboflavin production in large scale. 
393

394 Figure 6. The BsRF::ribDG-sRNA strain is stable and highly productive in a bioreactor scale. A

$3955 \mathrm{~L}$-batch was carried out for $52 \mathrm{~h}$ at $300 \mathrm{rpm}, 0,5 \mathrm{vvm}$ air supply, and $\mathrm{pH}$ controlled at $7.0 \pm 0.1$.

396 Samples were withdrawn periodically for sucrose (gray squares), riboflavin (orange circles), and

397 biomass (blue triangles) quantification. Data are presented as mean $\pm \operatorname{SD}(n=2)$. Raw data available in Supplementary Data 3.

\section{Discussion}

Previously underestimated, RNA has emerged in the last decade as a versatile tool to engineer strains and regulatory circuits (Chappell et al., 2015b; Kelly et al., 2018; Leistra et al., 2019; McCarty et al., 2020). When it comes to regulating gene expression, sRNA stands up as an efficient and easy-to-engineer tool. It has been intensely used to engineer bacteria for gene knockdown by preventing translation initiation (HoynesO'Connor and Moon, 2016; Liu et al., 2014; Na et al., 2013; Noh et al., 2017; Yang et al., 2019). Activation of gene expression has been achieved by engineering two RNA molecules, the sRNA and a toehold switch (for translation initiation control) or a Sense target RNA (for transcription termination control) (Chappell et al., 2015a; Green et al., 2014). Our strategy differs from the latter by targeting intrinsic regulatory RNAs (riboswitches) with synthetic sRNAs to activate gene expression.

Synthetic sRNAs had been previously designed to target the riboswitches $m e t H, x p t$, and pbuE. The sRNAs targeting the metH and $x p t$ riboswitches completely failed, and the

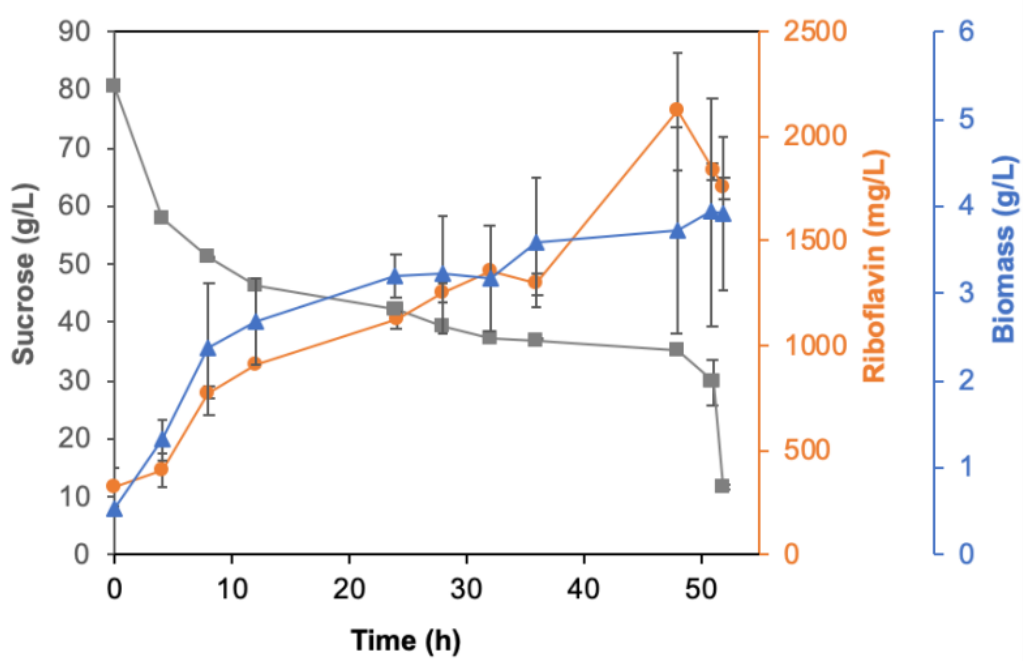


414 sRNA targeting the pbuE riboswitch only activated gene expression by 3.1 -fold (Chappell

415 et al., 2015a). Further improvement of the pbuE sRNA elevated the activation to 13.4-

416 fold (Meyer et al., 2016). We have successfully designed sRNAs targeting both the xpt

417 and the pbuE riboswitches. Our pbuE-sRNA displays a 103-fold gene activation, which

418 is much higher than achieved by others. Differently from others, our sRNA works as single genome copy. Using genome integrated sRNA genes, instead of plasmid-borne,

420 favors strain stability for engineering metabolite producers suitable for large scale

421 cultivation.

422 We used the riboswitch-targeting sRNAs to engineer a riboflavin-producing strain. The resulting strains were able to reach $50 \%$ increase in the riboflavin titer using one sRNA and $65 \%$ increase using two sRNAs. Targeting riboswitches with sRNAs seems more efficient than permanent edition. Recently, the ribDG riboswitch and the purR gene have been edited through CRISPR-Cas9 with the same goal of engineering a riboflavinproducing strain. The engineered strain produced $44 \%$ more riboflavin than the parental one (Boumezbeur et al., 2020). Interestingly, the study reports a similar improvement in culture density as observed here. Achieving similar growth improvements using different tools indicates that there are non-anticipated growth-limiting levels of gene expression within the purine and riboflavin pathways that have been alleviated by both approaches.

432 We have developed a new synthetic sRNA tool that targets bacterial riboswitches and activates gene expression at the transcription level. The tool has been validated in vitro and in vivo against different targets demonstrating specificity. Furthermore, we used the riboswitch-targeting sRNAs to efficiently engineer a riboflavin-producing strain. Finally, we developed an easy-to-engineer multiplex sRNA to target up to five different genes simultaneously. Multiplex sRNA overcomes the obstacles faced by protein-based

438 multiplex control. The tools we developed are broadly applicable to bacterial 439 riboswitches, and are useful to modulate expression of essential genes that cannot be 440 knocked out. 


\section{Acknowledgements}

443 This work was supported by the São Paulo Research Foundation (FAPESP) [grant

444 2014/17564-7 and 2020/08699-7]; Conselho Nacional de Desenvolvimento Científico e

445 Tecnológico (CNPq) [grant 290110/2017-3 and INCT BioSyn]; and Coordenação de

446 Aperfeiçoamento de Pessoal de Nível Superior - Brasil (CAPES) [Finance Code 001].

447

448 Supplementary files

449 Supplementary material

450

451 Mendeley data

452 https://data.mendeley.com/datasets/84yybmrtxx/1

453 DOI: $10.17632 / 84 y y b m r t x x .1$

454 Supplementary data 1

455 Supplementary data 2

456 Supplementary data 3

457

458 


\section{References}

460 Bacher, A., Eisenreich, W., Kis, K., Ladenstein, R., Richter, G., Scheuring, J., Weinkauf, S., 1993. Biosynthesis of Flavins, in: Dugas, H., Schmidtchen, F.P. (Eds.), Bioorganic Chemistry Frontiers. Springer Berlin Heidelberg, Berlin, Heidelberg, pp. 147-192. https://doi.org/10.1007/978-3-642-78110-0_5

Boumezbeur, A.H., Bruer, M., Stoecklin, G., Mack, M., 2020. Rational engineering of transcriptional riboswitches leads to enhanced metabolite levels in Bacillus subtilis. Metab Eng 61, 58-68. https://doi.org/10.1016/j.ymben.2020.05.002

Chappell, J., Takahashi, M.K., Lucks, J.B., 2015a. Creating small transcription activating RNAs. Nat Chem Biol 11, 214-220. https://doi.org/10.1038/nchembio.1737

Chappell, J., Watters, K.E., Takahashi, M.K., Lucks, J.B., 2015b. A renaissance in RNA synthetic biology: new mechanisms, applications and tools for the future. Curr Opin Chem Biol 28, 47-56. https://doi.org/https://doi.org/10.1016/j.cbpa.2015.05.018

Christiansen, L.C., Schou, S., Nygaard, P., Saxild, H.H., 1997. Xanthine metabolism in Bacillus subtilis: characterization of the xpt-pbuX operon and evidence for purineand nitrogen-controlled expression of genes involved in xanthine salvage and catabolism. J Bacteriol 179, 2540-2550. https://doi.org/10.1128/jb.179.8.25402550.1997

Correa, G.G., Lins, M.R.C.R., Silva, B.F., de Paiva, G.B., Zocca, V.F.B., Ribeiro, N.V., Picheli, F.P., Mack, M., Pedrolli, D.B., 2020. A modular autoinduction device for control of gene expression in Bacillus subtilis. Metab Eng. https://doi.org/10.1016/j.ymben.2020.03.012

483 Filonov, G.S., Moon, J.D., Svensen, N., Jaffrey, S.R., 2014. Broccoli: rapid selection of an RNA mimic of green fluorescent protein by fluorescence-based selection and directed evolution. J Am Chem Soc 136, 16299-16308. 
487 Green, A.A., Silver, P.A., Collins, J.J., Yin, P., 2014. Toehold switches: de-novo-

488 designed regulators of gene expression. Cell 159, 925-939.

489 https://doi.org/10.1016/j.cell.2014.10.002

490 Hoynes-O'Connor, A., Moon, T.S., 2016. Development of Design Rules for Reliable

491 Antisense RNA Behavior in E. coli. ACS Synth Biol 5, 1441-1454.

$492 \quad$ https://doi.org/10.1021/acssynbio.6b00036

493 Johansen, L.E., Nygaard, P., Lassen, C., Agerso, Y., Saxild, H.H., 2003. Definition of a

494 Second Bacillus subtilis pur Regulon Comprising the pur and xpt-pbuX Operons

495 plus pbuG, nupG (yxjA), and pbuE (ydhL). J Bacteriol 185, 5200-5209.

$496 \quad$ https://doi.org/10.1128/jb.185.17.5200-5209.2003

497 Kalvari, I., Nawrocki, E.P., Ontiveros-Palacios, N., Argasinska, J., Lamkiewicz, K.,

498 Marz, M., Griffiths-Jones, S., Toffano-Nioche, C., Gautheret, D., Weinberg, Z.,

499 Rivas, E., Eddy, S.R., Finn, R.D., Bateman, A., Petrov, A.I., 2021. Rfam 14:

500 expanded coverage of metagenomic, viral and microRNA families. Nucleic Acids

501 Res 49, D192-D200. https://doi.org/10.1093/nar/gkaa1047

502 Kelly, C.L., Harris, A.W.K., Steel, H., Hancock, E.J., Heap, J.T., Papachristodoulou, A., 503 2018. Synthetic negative feedback circuits using engineered small RNAs. Nucleic

$504 \quad$ Acids Res 46, 9875-9889. https://doi.org/10.1093/nar/gky828

505 Leistra, A.N., Curtis, N.C., Contreras, L.M., 2019. Regulatory non-coding sRNAs in

506 bacterial metabolic pathway engineering. Metab Eng 52, 190-214.

$507 \quad$ https://doi.org/https://doi.org/10.1016/j.ymben.2018.11.013

508 Lins, M.R.C.R., Correa, G.G., Amorim, L.A.S., Franco, R.A.L., Ribeiro, N.V., de Jesus,

509 V.N., Pedrolli, D.B., 2021. Characterization of five purine riboswitches in cellular

$510 \quad$ and cell-free expression systems. bioRxiv 2021.04.14.439898.

$511 \quad$ https://doi.org/10.1101/2021.04.14.439898

512 Liu, Y., Zhu, Y., Li, J., Shin, H., Chen, R.R., Du, G., Liu, L., Chen, J., 2014. Modular

513 pathway engineering of Bacillus subtilis for improved $\mathrm{N}$-acetylglucosamine

514 production. Metab Eng 23, 42-52. 
https://doi.org/https://doi.org/10.1016/j.ymben.2014.02.005

516 Lou, C., Stanton, B., Chen, Y.-J., Munsky, B., Voigt, C.A., 2012. Ribozyme-based

517 insulator parts buffer synthetic circuits from genetic context. Nat Biotechnol 30,

$518 \quad$ 1137. https://doi.org/10.1038/nbt.2401 https://www.nature.com/articles/nbt.2401\#supplementary-information

Mandal, M., Boese, B., Barrick, J.E., Winkler, W.C., Breaker, R.R., 2003. Riboswitches control fundamental biochemical pathways in Bacillus subtilis and other bacteria. Cell 113, 577-586.

Mandal, M., Breaker, R.R., 2004. Gene regulation by riboswitches. Nat Rev Mol Cell Biol 5, 451-463. https://doi.org/10.1038/nrm1403

Marcano-Velázquez, J.G., Batey, R.T., 2015. Structure-guided mutational analysis of gene regulation by the Bacillus subtilis pbuE adenine-responsive riboswitch in a cellular context. J Biol Chem 290, 4464-4475. https://doi.org/10.1074/jbc.M114.613497

Mars, R.A.T., Nicolas, P., Denham, E.L., van Dijl, J.M., 2016. Regulatory RNAs in Bacillus subtilis: a Gram-Positive Perspective on Bacterial RNA-Mediated Regulation of Gene Expression. Microbiol Mol Biol Rev 80, 1029 LP - 1057. https://doi.org/10.1128/MMBR.00026-16

McCarty, N.S., Graham, A.E., Studená, L., Ledesma-Amaro, R., 2020. Multiplexed CRISPR technologies for gene editing and transcriptional regulation. Nat Commun 11, 1-13. https://doi.org/10.1038/s41467-020-15053-x

Meyer, S., Chappell, J., Sankar, S., Chew, R., Lucks, J.B., 2016. Improving fold activation of small transcription activating RNAs (STARs) with rational RNA engineering strategies. Biotechnol Bioeng 113, 216-225. https://doi.org/https://doi.org/10.1002/bit.25693

540 Mironov, A.S., Gusarov, I., Rafikov, R., Lopez, L.E., Shatalin, K., Kreneva, R.A., 
https://doi.org/10.1016/s0092-8674(02)01134-0

544 Na, D., Yoo, S.M., Chung, H., Park, H., Park, J.H., Lee, S.Y., 2013. Metabolic

545 engineering of Escherichia coli using synthetic small regulatory RNAs. Nat

$546 \quad$ Biotechnol 31, 170-174. https://doi.org/10.1038/nbt.2461

547 Nielsen, A.A.K., Der, B.S., Shin, J., Vaidyanathan, P., Paralanov, V., Strychalski, E.A.,

548 Ross, D., Densmore, D., Voigt, C.A., 2016. Genetic circuit design automation. Science (80- ) 352, aac7341. https://doi.org/10.1126/science.aac7341

Noh, M., Yoo, S.M., Kim, W.J., Lee, S.Y., 2017. Gene Expression Knockdown by Modulating Synthetic Small RNA Expression in $<$ em>Escherichia coli</em $>$. Cell Syst 5, 418-426.e4. https://doi.org/10.1016/j.cels.2017.08.016

Pedrolli, D., Langer, S., Hobl, B., Schwarz, J., Hashimoto, M., Mack, M., 2015. The ribB FMN riboswitch from Escherichia coli operates at the transcriptional and translational level and regulates riboflavin biosynthesis. FEBS J 282. https://doi.org/10.1111/febs.13226

Pedrolli, D.B., Matern, A., Wang, J., Ester, M., Siedler, K., Breaker, R., MacK, M., 2012. A highly specialized flavin mononucleotide riboswitch responds differently to similar ligands and confers roseoflavin resistance to Streptomyces davawensis.

Radeck, J., Kraft, K., Bartels, J., Cikovic, T., Durr, F., Emenegger, J., Kelterborn, S., Sauer, C., Fritz, G., Gebhard, S., Mascher, T., 2013. The Bacillus BioBrick Box: generation and evaluation of essential genetic building blocks for standardized work with Bacillus subtilis. J Biol Eng 7, 29. https://doi.org/10.1186/1754-1611-7-

Richardson, S.M., Wheelan, S.J., Yarrington, R.M., Boeke, J.D., 2006. GeneDesign: rapid, automated design of multikilobase synthetic genes. Genome Res 16, 550556. https://doi.org/10.1101/gr.4431306

Rodrigo, G., Jaramillo, A., 2014. RiboMaker: computational design of conformationbased riboregulation. Bioinformatics 30, 2508-2510. 
https://doi.org/10.1093/bioinformatics/btu335

572 Rostain, W., Landrain, T.E., Rodrigo, G., Jaramillo, A., 2015. Regulatory RNA design

573 through evolutionary computation and strand displacement. Methods Mol Biol 1244, 63-78. https://doi.org/10.1007/978-1-4939-1878-2_4

Rouillard, J.-M., Lee, W., Truan, G., Gao, X., Zhou, X., Gulari, E., 2004. Gene2Oligo: oligonucleotide design for in vitro gene synthesis. Nucleic Acids Res 32, W176-80. https://doi.org/10.1093/nar/gkh401

578 Saxild, H.H., Brunstedt, K., Nielsen, K.I., Jarmer, H., Nygaard, P., 2001. Definition of the Bacillus subtilis PurR Operator Using Genetic and Bioinformatic Tools and Expansion of the PurR Regulon with glyA, guaC, pbuG, xpt-pbuX, yqhZ-folD, and pbuO. J Bacteriol 183, 6175-6183. https://doi.org/10.1128/jb.183.21.61756183.2001

Shi, T., Wang, Y., Wang, Z., Wang, G., Liu, D., Fu, J., Chen, T., Zhao, X., 2014. Deregulation of purine pathway in Bacillus subtilis and its use in riboflavin biosynthesis. Microb Cell Fact 13, 101. https://doi.org/10.1186/s12934-014-0101-8

Shimoni, Y., Friedlander, G., Hetzroni, G., Niv, G., Altuvia, S., Biham, O., Margalit, H., 2007. Regulation of gene expression by small non-coding RNAs: a quantitative view. Mol Syst Biol 3, 138. https://doi.org/https://doi.org/10.1038/msb4100181 small regulatory RNA expression platforms for rapid and multiplex gene expression knockdown. Metab Eng 54, 180-190. https://doi.org/https://doi.org/10.1016/j.ymben.2019.04.003

597 Yang, Y., Lin, Y., Li, L., Linhardt, R.J., Yan, Y., 2015. Regulating malonyl-CoA 
bioRxiv preprint doi: https://doi.org/10.1101/2021.06.21.449321; this version posted June 21, 2021. The copyright holder for this preprint

(which was not certified by peer review) is the author/funder, who has granted bioRxiv a license to display the preprint in perpetuity. It is made available under aCC-BY-NC-ND 4.0 International license.

599 products. Metab Eng 29, 217-226.

$600 \quad$ https://doi.org/https://doi.org/10.1016/j.ymben.2015.03.018

601 Zadeh, J.N., Steenberg, C.D., Bois, J.S., Wolfe, B.R., Pierce, M.B., Khan, A.R., Dirks,

602 R.M., Pierce, N.A., 2011. NUPACK: Analysis and design of nucleic acid systems.

603

J Comput Chem 32, 170-173. https://doi.org/10.1002/jcc.21596

604 